\title{
Quark Mass Dependence of the QCD Equation of State on $N_{\tau}=8$ Lattices
}

Wolfgang Söldner* for the RBC-Bielefeld and hot $Q C D$ collaborations ${ }^{\dagger}$

Physics Department, Brookhaven National Laboratory, Upton, NY 11973, USA

E-mail: soeldner@bnl.gov

\begin{abstract}
We currently perform calculations with an improved staggered fermion action (p4fat3). We use a strange quark mass that has been tuned to its physical value and light quarks of mass $m_{s} / 20$ on lattices of size $32^{3} \times 8$. This corresponds to an almost physical light quark mass. We present first results on the low temperature part of the equation of state of QCD. Through comparison with the preliminary hotQCD results on the $N_{\tau}=8$ equation of state, which have been obtained with twice heavier light quark masses, we can quantify the quark mass dependence of the equation of state in the low temperature regime. We also comment on the quark mass dependence of the equation of state at high temperature.
\end{abstract}

The XXVI International Symposium on Lattice Field Theory

July 14-19 2008

Williamsburg, Virginia, USA

\footnotetext{
* Speaker.

${ }^{\dagger}$ This work has been supported in part by contract DE-AC02-98CH10886 with the U.S. Department of Energy
} 


\section{Introduction}

One major topic in thermodynamics of QCD and, especially, lattice QCD is the calculation the Equation of State (EoS). While there has been great progress in the recent years still several issues remain unresolved. One of them is the observed deviation in the EoS of the lattice computation compared to what one expects from a Hadron Resonance Gas model (HRG). As can be seen on the right hand side of Fig. 1 the HRG description shows deviations from the lattice QCD data [1], especially in the low temperature regime. On the contrary, because the HRG model is in quite good agreement with experiment in the low temperature region [2], one would expect that the HRG model also gives a good description for the low temperature region of the lattice QCD equation of state. A possible source for the discrepancy, aside from lattice discretization errors which still may
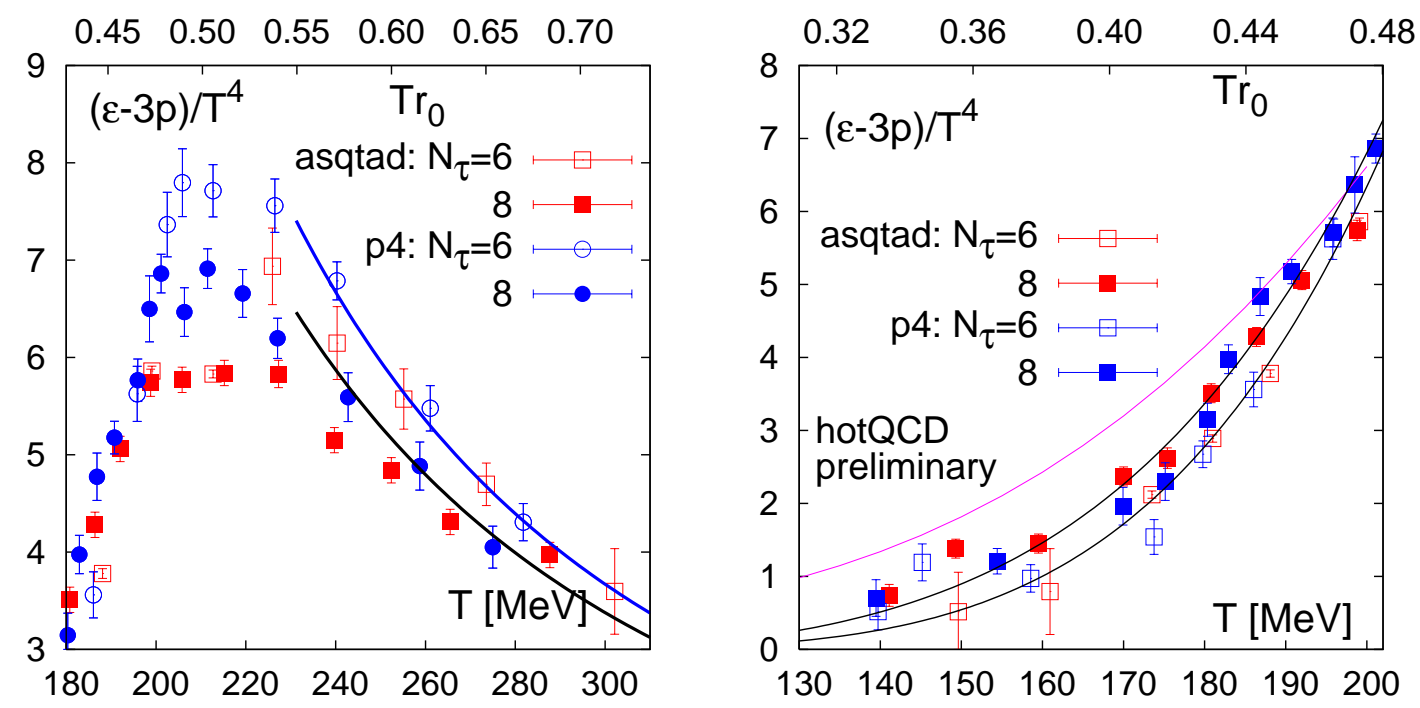

Figure 1: Trace anomaly from lattice QCD at various $N_{\tau}$ for $m_{q}=0.1 m_{s}$ (left). Deviations are observed between HRG and lattice QCD for the low temperature regime (right).

play a role on the $N_{\tau}=8$ lattices, may be due to the fact that previous lattice studies of the EoS had been performed with light quark mass values which are about a factor two larger than in nature. This corresponds to a light pseudo-scalar mass of about $220 \mathrm{MeV}$. In order to quantify the effects of too large light quark masses we present new calculations for the lattice QCD equation of state at a smaller light quark mass $m_{q}=0.05 m_{s}$ compared to our earlier calculation with $m_{q}=0.1 m_{s}$ where $m_{s}$ is the strange quark mass.

The new data for $m_{q}=0.05 m_{s}$ also is used to investigate the properties of the chiral phase transition. In particular, we will present results on the scaling with the light quark mass of the chiral condensate and the chiral susceptibility which is relevant for identifying the critical point of the chiral phase transition.

The paper is organized as follows. In Sec. 2 we give an overview of numerical details and outline the calculation of the EoS on the lattice. In Sec. 3 the calculation of the potential and the change in the scale for the $m_{q}=0.05 m_{s}$ case is discussed. The results for the EoS with $m_{q}=0.05 m_{s}$ are presented in Sec. 4 and results for the chiral condensate and susceptibility are shown in Sec. 5. 


\section{Numerical Details and Setup}

The computation of the QCD equation of state on the lattice requires large resources. Our calculations have been performed on IBM BlueGene/L and QCDOC supercomputers at Lawrence Livermore National Laboratory, the New York Center for Computational Sciences (NYCCS), and Jülich Supercomputing Centre. For our calculations we use the p4fat 3 action and the RHMC algorithm [1]. The computational details are summarized in Tab. 1 and Ref. [3]. Further information can be found in Refs. [4, 5, 6].

\begin{tabular}{lll} 
Masses: & $m_{q}=0.1 m_{s}$ & $m_{q}=0.00081-0.00370$ \\
& $m_{q}=0.05 m_{s}$ & $m_{q}=0.00120-0.00145$ \\
\hline Pion Masses: & $m_{q}=0.1 m_{s}$ & $m_{\pi} \approx 220 \mathrm{MeV}$ \\
& $m_{q}=0.05 m_{s}$ & $m_{\pi} \approx 160 \mathrm{MeV}$ \\
\hline Volume $(T \neq 0):$ & $m_{q}=0.1 m_{s}$ & $32^{3} \times 8$ \\
& $m_{q}=0.05 m_{s}$ & $32^{3} \times 8$ \\
\hline Volume $(T=0):$ & $m_{q}=0.1 m_{s}$ & $32^{4}$ \\
& $m_{q}=0.05 m_{s}$ & $32^{4}$ \\
\hline Stats. $(T \neq 0):$ & $m_{q}=0.1 m_{s}$ & $\# 8,000-37,000$ \\
& $m_{q}=0.05 m_{s}$ & $\# 5,000-20,000$ \\
\hline Stats. $(T=0):$ & $m_{q}=0.1 m_{s}$ & $\# 2,000-6,000$ \\
& $m_{q}=0.05 m_{s}$ & $\# 1,500-2,000$ \\
\hline
\end{tabular}

Table 1: Overview of the simulation details.

In the following we give a brief sketch of the calculation of the EoS on the lattice. The basic quantity is the trace anomaly $\Theta^{\mu \mu} \equiv \varepsilon-3 p$ with the energy-momentum tensor $\Theta^{\mu v}$, energy density $\varepsilon$, and pressure $p$. The trace anomaly can be computed on the lattice in terms of the action density, and the strange and light quark chiral condensates. We are only interested in the thermal part of the trace anomaly and subtract the zero temperature part,

$$
\Theta^{\mu \mu} \equiv \varepsilon-3 p=(\varepsilon-3 p)_{T}-(\varepsilon-3 p)_{T=0}
$$

From this expression we can determine the pressure through the thermodynamic relation $\Theta^{\mu \mu} / T^{4}=$ $T \partial\left(p / T^{4}\right) / \partial T$ which is integrated to yield

$$
\frac{p(T)}{T^{4}}-\frac{p\left(T_{0}\right)}{T_{0}^{4}}=\int_{T_{0}}^{T} \mathrm{~d} \bar{T} \frac{1}{\bar{T}^{5}} \Theta^{\mu \mu}(\bar{T}) .
$$

Here $T_{0}$ is chosen to be in the deep hadronic region where $p\left(T_{0}\right)$ is already exponentially small. The energy density $\varepsilon$ then is determined given $\Theta^{\mu \mu}$ and $p$. For further details see Ref. [1].

\section{Potential}

To account for the quark mass dependence of the lattice cut-off, which corresponds to a shift in the temperature scale when we lower the light quark mass, we have calculated the potential for 


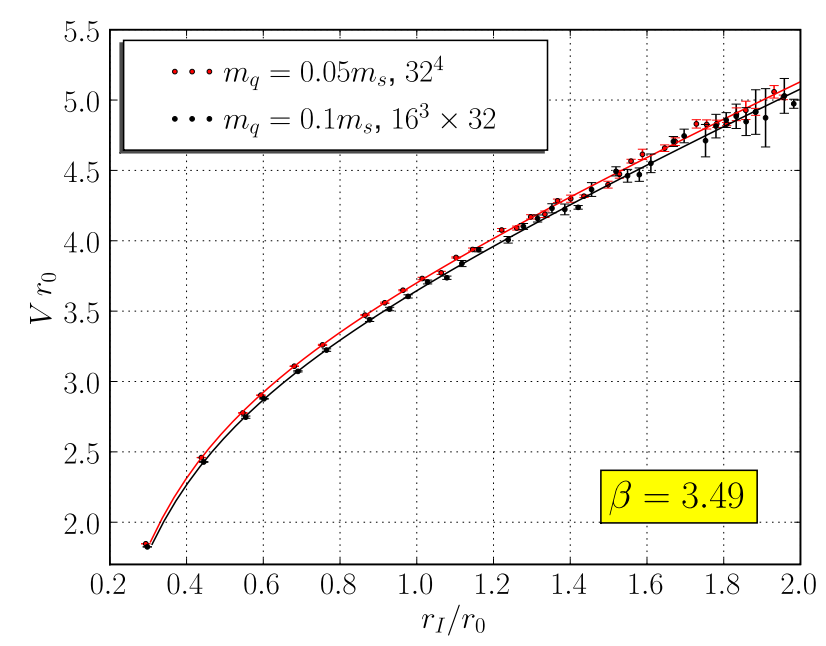

Figure 2: Potential $V(r)$ vs. improved distance $r_{I}$ at $\beta=3.49$ for $m_{q}=0.05 m_{s}$ and $m_{q}=0.1 m_{s}$. Both quantities are given in dimensionless units using the Sommer scale parameter $r_{0}$.

several values of $\beta$. For $m_{q}=0.1 m_{s}$ the scale was calculated on $16^{3} \times 32$ lattices, see Ref. [1]. In the case of $m_{q}=0.05 m_{s}$ we computed the potential on $32^{4}$ lattices. We have set $r_{0}=0.469 \mathrm{fm}$. The results and some simulation details are summarized in Tab. 2 where we also compare lattice scales obtained for $m_{q}=0.05 m_{s}$ to $m_{q}=0.1 m_{s}$. For illustration we plotted the potential for $\beta=3.49$ in Fig. 2 both for $m_{q}=0.05 m_{s}$ and $m_{q}=0.1 m_{s}$.

From the preliminary results in Tab. 2 we conclude that within error bars the scale determined for $m_{q}=0.05 m_{s}$ is consistent with the older results for $m_{q}=0.1 m_{s}$. We are planning to increase statistics and include more $\beta$ values in the future. For the moment we will make use of the global mass fit [7]

$$
a_{m_{q}=0.05 m_{s}}=\exp \left(-2 A \Delta m_{q}\right) a_{m_{q}=0.1 m_{s}},
$$

in order to determine the scale. The value for $A$ is found to be $A=1.40(3)$. In this fit more data points are involved than in our current analysis for $m_{q}=0.05 m_{s}$. Using this fit we find a shift in

\begin{tabular}{lllll}
$\beta$ & 3.49 & 3.51 & 3.53 & 3.54 \\
\hline$a_{m_{q}=0.1 m_{s}}[\mathrm{fm}]$ & 0.1455 & 0.1370 & & 0.1272 \\
$a_{m_{q}=0.05 m_{s}}[\mathrm{fm}]$ & 0.1435 & 0.1385 & 0.1306 & \\
\hline$r_{0} / a_{m_{q}=0.1 m_{s}}$ & $3.223(23)$ & $3.423(30)$ & & $3.689(37)$ \\
$r_{0} / a_{m_{q}=0.05 m_{s}}$ & $3.2675(90)$ & $3.386(20)$ & $3.592(11)$ & \\
\hline$T_{m_{q}=0.1 m_{s}}[\mathrm{MeV}]$ & $169.5(1.2)$ & $180.0(1.6)$ & & $194.0(1.9)$ \\
$T_{m_{q}=0.05 m_{s}}[\mathrm{MeV}]$ & $171.85(47)$ & $178.1(1.1)$ & $188.90(60)$ & \\
\hline$\#_{m_{q}=0.1 m_{s}}$ & 330 & 140 & & 300 \\
$\#_{m_{q}=0.05 m_{s}}$ & 200 & 200 & 170 & \\
\hline
\end{tabular}

Table 2: Results and overview for the calculation of the potential. The last two rows give the number of configurations we have used in our analysis. 
temperature of about $0.5 \mathrm{MeV}$.

\section{Mass Dependence of the QCD Equation of State}

In Fig. 3 we plot our result for the trace anomaly for $m_{q}=0.05 m_{s}$. We compare to the $m_{q}=$ $0.1 m_{s}$ case from Ref. [1,3] as well as to the HRG model result. The curves in the plot correspond to fits with a quadratic ansatz in $T$ which fits the data quite well. Note that for our calculation of the trace anomaly for the HRG all resonances were chosen to be at their physical mass. We also note that for the $m_{q}=0.05 m_{s}$ case the lightest pseudo-scalar mass ${ }^{1}$ is about $160 \mathrm{MeV}$ which is very close to its physical value. The shift of about $0.5 \mathrm{MeV}$ in the scale, as discussed in Sec. 3, for the $m_{q}=0.05 m_{s}$ case has been taken into account.

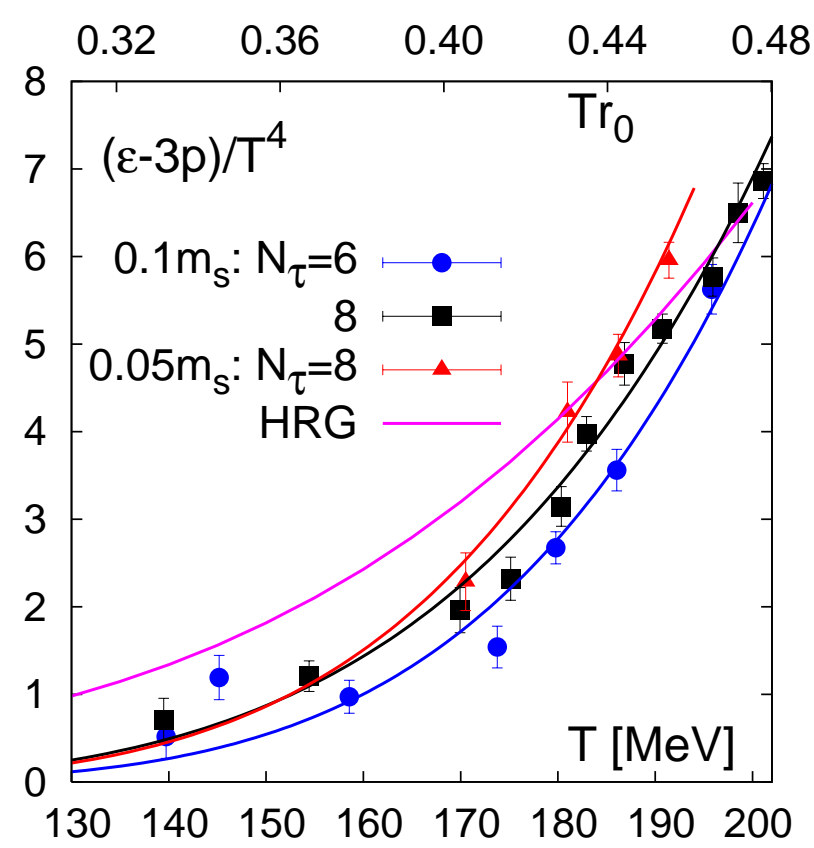

Figure 3: Trace anomaly for $m_{q}=0.05 m_{s}$ compared to the HRG and $m_{q}=0.1 m_{s}$ results. The curves are $T^{2}$ fits to the corresponding data made to highlight the cut-off and quark mass dependence of the various data sets.

From Fig. 3 we observe in the transition region a shift towards lower temperature for $(e-$ $3 p) / T^{4}$ for $m_{q}=0.05 m_{s}$ of about $4 \mathrm{MeV}$ compared to $m_{q}=0.1 m_{s}$ (with $N_{\tau}=8$ ). For the larger temperature regime we find that for the smaller quark mass there is quite good agreement with the HRG model. However, for the lower temperature region we observe deviations from the HRG in that case. We will increase the statistics and add more data points in the lower temperature region in the future in order to clarify the situation.

\footnotetext{
${ }^{1}$ Note that within the staggered formalism only one light pseudo-scalar exists at finite values of the lattice cut-off. Full flavor symmetry is only recovered in the continuum limit.
} 


\section{Chiral Condensate}

In this section we present new results on the chiral condensate and its susceptibility. We consider a combination of the light and strange quark condensate to eliminate quadratic divergencies with respect to the additive quark mass renormalization

$$
\Delta_{l, s}=\frac{\langle\bar{\psi} \psi\rangle_{l, \tau}-\frac{\hat{m}_{l}}{\hat{m}_{s}}\langle\bar{\psi} \psi\rangle_{s, \tau}}{\langle\bar{\psi} \psi\rangle_{l, 0}-\frac{\hat{m}_{l}}{\hat{m}_{s}}\langle\bar{\psi} \psi\rangle_{s, 0}}
$$

We normalize this combination by its zero temperature value to cancel the multiplicative renormalization factor. In Fig. 4 we show on the left hand side results for $m_{q}=0.1 m_{s}$ with $N_{\tau}=6,8$ as
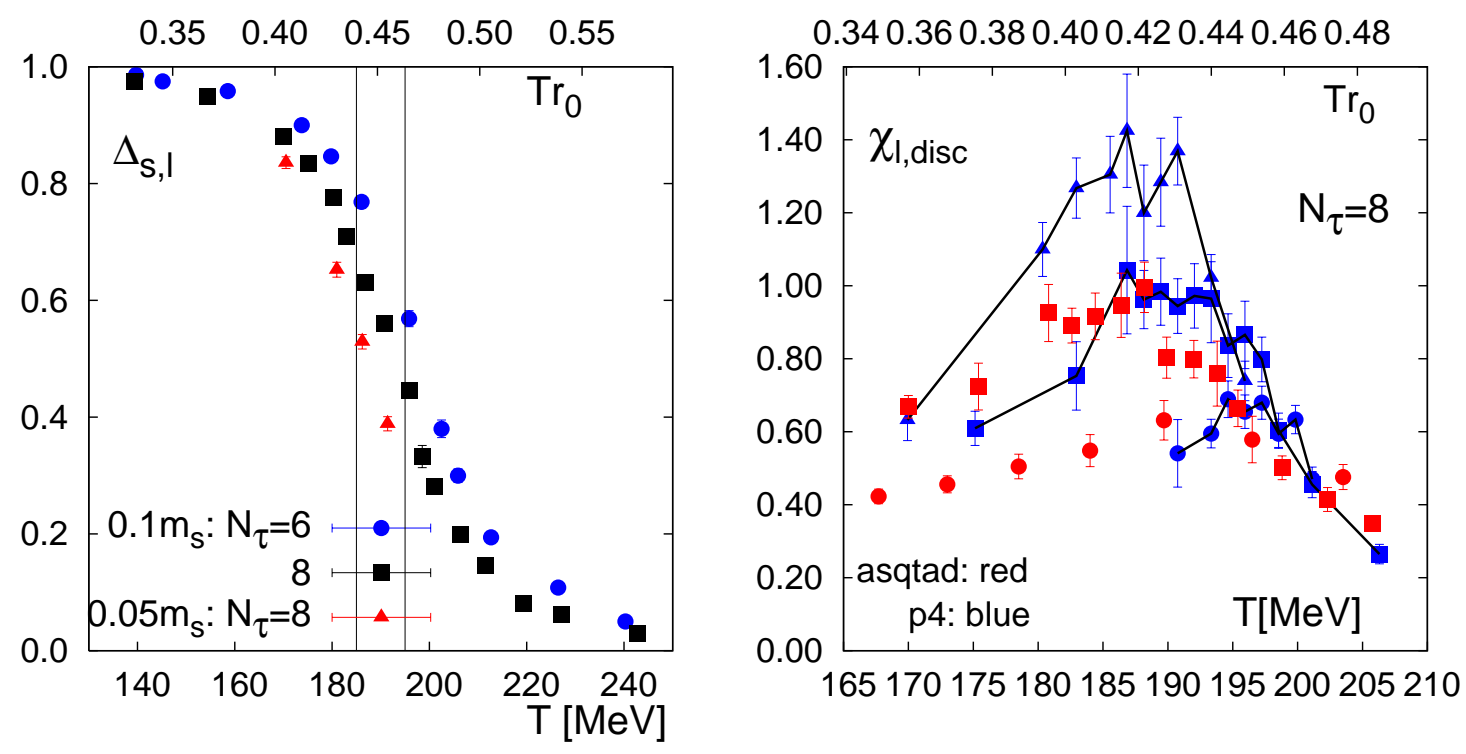

Figure 4: We plot $\Delta_{l, s}$ for the two different light quark masses (left). The disconnected light chiral susceptibility is shown on the right hand side for light quark masses $m_{q}=0.2 m_{s}$ (circles), $m_{q}=0.1 m_{s}$ (squares), and $m_{q}=0.05 m_{s}$ (triangles).

well as results for $m_{q}=0.05 m_{s}$ with $N_{\tau}=8$. We observe a sharp drop of $\Delta_{l, s}$ in the region of $T_{c}$ for both data sets with $m_{q}=0.1 m_{s}$ and $m_{q}=0.05 m_{s}$. We note that we will present data for more different light quark masses in the future which will allow us to probe the limit $m_{q} \rightarrow 0$. With only two different light quark masses we cannot investigate this limit at the moment since near $T_{c}$ terms proportional to $m_{q}$ as well as $\sqrt{m_{q}}$ are present.

On the right hand side of Fig. 4 we plotted the disconnected light chiral susceptibility $\chi_{l, d i s c}$ for different light quark masses. We find that $\chi_{l, d i s c}$ shows a strong quark mass dependence over a wide temperature range. This can be understood as an increase in the fluctuations of Goldstone modes below $T_{c}$. The transition temperature should then be found near the right edge of the peak. Further evidence for this picture is given by the observation that the height of the peak of $\chi_{l, d i s c}$ scales as $1 / \sqrt{m_{q}}$. 


\section{Summary}

We presented new data for the equation of state for $m_{q}=0.05 m_{s}$ close to the transition temperature. The pion mass is as low as $\approx 160 \mathrm{MeV}$. We compared the trace anomaly to earlier results for $m_{q}=0.1 m_{s}$. A total shift of the transition region of about $4 \mathrm{MeV}$ is found towards smaller temperatures. About $0.5 \mathrm{MeV}$ of that shift can be contributed to the shift in the scale. Comparing to the Hadron Resonance Gas model (HRG) we find that at larger temperatures the results for $m_{q}=0.05 m_{s}$ are consistent with HRG model while there are deviations at lower temperatures. Calculations at different temperatures are currently ongoing.

We have studied the behavior of $\Delta_{l, s}$ for the two different quark masses. A sharp drop in the vicinity of the critical temperature is observed. Furthermore, we have presented results for the disconnected light chiral susceptibility. In the transition region $\chi_{l, d i s c}$ shows a broad peak for the different light quark masses which rises as $1 / \sqrt{m_{q}}$. This gives support for a picture where the Goldstone modes are causing the fluctuations below $T_{c}$.

\section{Acknowledgments}

We are grateful to LLNL, NNSA, New York Center for Computational Sciences, and the Jülich Supercomputing Centre for providing access to the BlueGene/L supercomputers.

\section{References}

[1] M. Cheng et al., "The QCD Equation of State with almost Physical Quark Masses," Phys. Rev. D 77, 014511 (2008) [arXiv:0710.0354 [hep-lat]].

[2] P. Braun-Munzinger, K. Redlich and J. Stachel, arXiv:nucl-th/0304013; A. Andronic, P. Braun-Munzinger and J. Stachel, Nucl. Phys. A 772, 167 (2006) [arXiv:nucl-th/0511071].

[3] R. Gupta, "The EOS from simulations on BlueGene L Supercomputer at LLNL and NYBlue", PoS LAT2008 170 (2008)

[4] C. DeTar, "Recent Progress in Lattice QCD Thermodynamics“, PoS LAT2008 (2008)

[5] F. Karsch, "Recent lattice results on finite temperature and density QCD“, PoS LAT2007 015 (2007).

[6] C. DeTar, R. Gupta [HotQCD Collaboration], "Towards a precise determination of Tc with 2+1 flavors of quarks“, PoS LAT2007 179 (2007) [arXiv:hep-lat/0710.1655].

[7] M. Cheng et al., Phys. Rev. D 74, 054507 (2006) [arXiv:hep-lat/0608013]. 